\section{PERIODONTOLOGY; EPIDEMIOLOGY}

\section{Tobacco use and incidence of tooth loss among US male health professionals}

Dietrich T, Maserejian NN et al. J Dent Res 2007; 86: 373-377

There was a dose-related effect of smoking on tooth loss.

The effects of smoking on periodontal health are well-established, but data on dose related to tooth loss are sparse. This study examined longitudinal data from 51,529 US male health professionals (58\% dentists) starting in 1986 (aged 40-75 yrs). Some $90 \%$ responded to questionnaires each 2 years, and in 2002, after exclusions, data were included from 43,112 subjects (61\% dentists).

At baseline, 9\% were current, and 44\% former, smokers. In 2002, compared with never-smokers in a model with adjustment for numerous potential confounders, significant hazard ratios (HRs) for risk of tooth loss were respectively: for smokers who had quit 10+ yrs earlier, 1.2; 6-9 yrs, 1.5; 3-5 yrs, 1.6; 1-2 yrs, 1.9; and <1 yr, 2.6. In current smokers, significant HRs were respectively: for smoking 1-4 cigarettes/day, $1.4 ; 5-14,1.9 ; 15-24,2.3 ; 25-34,2.7 ; 35-44,2.9$; and $45+, 3.0$.

DOI: $10.1038 / b d j .2007 .684$

\section{PREVENTIVE DENTISTRY}

Effect of three different dental health preventive programmes on young adult individuals: a randomized, blinded, parallel group, controlled evaluation of oral hygiene behaviour on plaque and gingivitis

Hugoson A, Lundgren D et al. J Clin Periodontol 2007; 34: 407-415

Preventive instruction was effective, but professional tooth cleaning did not affect results.

Dental disease may be reduced by various preventive approaches. In this 3 yr Swedish study of 400 patients aged 20-27 yrs from a public clinic and a private practice, they were randomised to 4 groups: controls; a personalised preventive programme for individuals at 2 month intervals with additional professional tooth cleaning for a random sub-group; another individual preventive programme; and the same programme given to groups of people.

The control group had significantly more gingivitis at $3 \mathrm{yrs}$, but differences between test groups were NS. Gingival health at baseline best predicted a healthy outcome. Participation in a preventive programme and knowledge of dental diseases were also predictors.

DOI: 10.1038/bdj.2007.685

\section{BEHAVIOURAL SCIENCE; EPIDEMIOLOGY}

Relationship between cognitive impairment and oral health: results of the Health 2000 Health Examination Survey in Finland

Syrjälä A-MH, Ylöstalo P et al. Acta Odont Scand 2007; 65: 103-108

These patients had poorer dental conditions, but there may be several explanations for the association.

Impairment of cognitive health $(\mathrm{CH})$ is increasing with longevity. This study investigated the relationship of $\mathrm{CH}$ and oral health $(\mathrm{OH})$ in a nationally representative sample of 2,320 Finns aged $55+$ yrs.

Subjects with good $\mathrm{CH}$ had significantly more teeth, less caries, fewer dentures, fewer denture problems and better denture hygiene. Those with moderate or severe $\mathrm{CH}$ impairment were 5 times more likely to have no teeth and no dentures.

The authors note that the association of poor $\mathrm{CH}$ and $\mathrm{OH}$ may relate to a lack of preventive measures, effects of medication, difficulties in treatment, levels of education and social networks. If $\mathrm{OH}$ is to improve in these patients, multifactorial support is needed. 